\title{
Prevalence of Pseudomonas aeruginosa in Various Clinical Samples and Its Antibiotic Susceptibility Pattern in a Tertiary Care Hospital
}

\author{
Sarada Dasari, H. Ravichandra Prakash ${ }^{*}$, C. Surya Kumari and D. Sisira \\ Department of Microbiology, Viswa Bharathi Medical College, India \\ *Corresponding author
}

\section{A B S T R A C T}

\begin{tabular}{|l|}
\hline Ke y w o r d s \\
Antibiotic \\
sensitivity, \\
antibiotic \\
resistance, \\
Pseudomonas \\
aeruginosa
\end{tabular}

Pseudomonas aeruginosa is one of the commonly isolated gram negative bacillus from various clinical samples. It is a commensal in healthy individuals but can be opportunistic pathogen especially in hospitalised patients. It is intrinsically resistant to many antibiotics. Hence the present study was undertaken to study the prevalence and antibiotic sensitivity pattern in our hospital. Various clinical samples like pus, sputum, urine, vaginal swab, pleural fluid, ear swabs and tracheal aspirate received in the microbiology laboratory were included in the study. Identification and antibiotic sensitivity testing was done by standard laboratory procedures. A total of 157 Pseudomonas aeruginosa were isolated from 858 clinical samples with a prevalence rate of $18.3 \%$. Age and gender wise distribution shows higher prevalence rate in 40-60 years age group and in males. Maximum isolates were from pus, sputum and urine samples. Antibiotics tested were ceftazidime, ceftriaxone, piperacillin-tazobactum, aztreonam, amikacin, gentamycin, netilmicin, ciprofloxacin, ofloxacin, meropenem, cefixime. Antibiotic sensitivity pattern was found to show higher sensitivity to meropenem, piperacillin-tazobactum and amikacin. Resistance was more pronounced in cefixime, netilmicin, ceftriaxone and aztreonam. Spread of drug resistance in Pseudomonas aeruginosa is to be prevented by using antibiotics judiciously adhering to the antibiotic policy.

\section{Introduction}

Pseudomonas aeruginosa is the most commonly isolated gram negative species which is not a member of family enterobacteriaceae $^{1}$. In the last two decades it has been more commonly implicated as aetiological agent in many infections in hospitalised patients with impaired immune defence mechanism ${ }^{2}$. It is ubiquitous in nature and is capable of causing human infections in many ways. It is aerobic, gram negative, motile bacilli. It is nonfermentative, utilises glucose oxidatively. It was called as blue pus due to pigment produced by it.

It has been implicated as causative agent in various infections like suppurative otitis media, urinary tract infections, orthopaedic infections, burns, surgical site infections, respiratory tract infections etc.

It is the $2^{\text {nd }}$ most common cause of ventilator associated pneumonia ${ }^{3}$. 
In critically ill patients Pseudomonas aeruginosa contributes $3 \%-15 \%$ of bloodstream infections with high mortality rate of about $27 \%-48 \%$. In spite of recent advances in treatment modalities, bacteremia due to Pseudomonas aeruginosa remains fatal in more than $20 \%$ of cases ${ }^{4}$.

Pseudomonas aeruginosa can survive difficult environmental conditions and displays intrinsic resistance to a wide variety of antimicrobial agents that promote organisms capacity to survive in hospital setting ${ }^{1}$. It possesses many drug resistant plasmids which confer resistance to several antibiotics. Most of the strains produce $\beta$ lactamases such as extended spectrum $\beta$ lactamases, carbapenems and Amp C $\beta$ lactamases ${ }^{5}$.

Due to increasing multi drug resistance in Pseudomonas aeruginosa available therapeutic options are decreasing. Areawise studies on antimicrobial susceptibility profiles are essential to guide policy on the appropriate use of antibiotics ${ }^{6}$.The present study was undertaken to know the prevalence of Pseudomonas aeruginosa and its antibiotic profile in our setting.

\section{Materials and Methods}

The present study was conducted in a tertiary care centre during the period March 2018 April 2019. Various clinical samples both from inpatients and outpatients sent to microbiology laboratory were included in the study. A total of 858 samples including pus, sputum, urine, vaginal swab, pleural fluid, ear swabs and tracheal aspirate were processed by conventional microbiological methods.

All the samples were inoculated on nutrient agar, blood agar, MacConkey agar and incubated overnight at $37^{\circ} \mathrm{C}$. Gram staining was done from samples as well as from culture which showed Gram negative bacilli. Characteristic sweety odour, pigment production on nutrient agar, non-lactose fermenting colonies on MacConkey agar, $\beta$ hemolytic colonies on blood agar were seen.

Isolates were confirmed as Pseudomonas aeruginosa by biochemical reactions. Oxidase and catalase tests were positive, nitrates were reduced to nitrites, indole production was negative, urease negative, citrate positive. TSI showed ALK slant/ no reaction in butt. All the isolates were motile by hanging drop method $^{7}$.

Antibiotic susceptibility pattern of Pseudomonas aeruginosa isolates was determined by Kirby-Bauer disc diffusion method on Mueller Hinton agar according to CLSI guidelines ${ }^{8}$.

Antibiotic discs obtained from Himedia were used. Antibiotics tested were ceftazidime (30 $\mu \mathrm{g})$, ceftriaxone $(30 \mu \mathrm{g})$, cefixime $(5 \mu \mathrm{g})$, piperacillin-tazobactum $\quad(100 / 10 \quad \mu \mathrm{g})$, Aztreonam (30 $\mu \mathrm{g})$, Amikacin (30 $\mu \mathrm{g})$, Gentamicin $(10 \mu \mathrm{g})$, Ciprofloxacin $(5 \mu \mathrm{g})$, Ofloxacin $(5 \mu \mathrm{g})$, meropenem $(\mu \mathrm{g})$, netilmicin $(\mu \mathrm{g})$, cefotaxime $(30 \mu \mathrm{g})$. Size of zone of inhibition was measured and susceptibility interpreted.

\section{Results and Discussion}

In the present study 157 (18.3\%) isolates of Pseudomonas aeruginosa were isolated from 858 samples in the duration of 1 year.

Out of 157 isolates $89(56.68 \%)$ were from males and $68(43.31 \%)$ were from females.

Age wise distribution shows maximum isolates were from 41-60 years age group and least number of isolates were from less than 20 years age group. 
Among the various samples included in the present study maximum of 58 (22.48\%) isolates were from pus, followed by sputum and urine (Table 1).

Agewise distribution shows maximum isolates were from 41-60 years age group and minimum from $<20$ years group. Isolation of Pseudomonas aeruginosa was higher in males $(56.68 \%)$ when compared with females $(43.31 \%)$ (Table 2).

Antibiotic sensitivity testing shows maximum sensitivity to Meropenem, Piperacillin-
Tazobactum and Amikacin. Higher resistance was shown by cefixime, ceftriaxone and netilmicin (Table 3). The present study was done in a tertiary care hospital to study the prevalence of Pseudomonas aeruginosa in various clinical samples and their sensitivity pattern.

In the present study Pseudomonas aeruginosa was isolated from 157 samples $(18.3 \%)$ out of a total of 858 samples. Similar rate of prevalence was observed in other studies, $17.05 \%$ prevalence was shown by Chander et al., ${ }^{9}, 24 \%$ by SarojGolia et al., ${ }^{10}$.

Table.1 Pseudomonas aeruginosa isolated from various clinical samples

\begin{tabular}{|l|l|l|l|}
\hline Sample & Number & Positive & Percentage \\
\hline Pus & 253 & 58 & $22.92 \%$ \\
\hline Sputum & 243 & 51 & $20.98 \%$ \\
\hline Urine & 275 & 41 & $14.90 \%$ \\
\hline Vaginal swab & 40 & 3 & $7.5 \%$ \\
\hline Pleural fluid & 22 & 2 & $9.09 \%$ \\
\hline Ear swab & 17 & 1 & $5.88 \%$ \\
\hline Tracheal aspirate & 8 & 1 & $12.5 \%$ \\
\hline Total & 858 & 157 & \\
\hline
\end{tabular}

Table.2 Age and genderwise distribution of Pseudomonas aeruginosa

\begin{tabular}{|c|c|c|c|}
\hline Age group & Male & Female & Total \\
\hline <20 years & 10 & 7 & $17(10.82 \%)$ \\
\hline 21-40 years & 19 & 16 & $35(22.29 \%)$ \\
\hline 41-60 years & 34 & 26 & $60(38.21 \%)$ \\
\hline >60 years & 26 & 19 & $45(28.66 \%)$ \\
\hline Total & $89(56.68 \%)$ & $68(43.31 \%)$ & 157 \\
\hline
\end{tabular}

Table.3 Antibiotic susceptibility pattern of Pseudomonas aeruginosa

\begin{tabular}{|c|c|c|c|}
\hline Sl. no & Antibiotics & Sensitive & Resistant \\
\hline $\mathbf{1 .}$ & Ceftazidime & $108(68.78 \%)$ & $49(31.21 \%)$ \\
\hline $\mathbf{2 .}$ & Ceftriaxone & $94(59.87 \%)$ & $63(40.12 \%)$ \\
\hline $\mathbf{3 .}$ & Cefixime & $51(32.48 \%)$ & $106(67.51 \%)$ \\
\hline $\mathbf{4 .}$ & Amikacin & $134(85.35 \%)$ & $23(14.64 \%)$ \\
\hline $\mathbf{5 .}$ & Gentamycin & $120(76.43 \%)$ & $37(23.56 \%)$ \\
\hline 6. & Netilmicin & $79(50.31 \%)$ & $78(49.68 \%)$ \\
\hline 7. & Ciprofloxacin & $105(66.87 \%)$ & $52(33.12 \%)$ \\
\hline $\mathbf{8 .}$ & Ofloxacin & $112(71.33 \%)$ & $45(28.66 \%)$ \\
\hline $\mathbf{9 .}$ & Aztreonam & $89(56.68 \%)$ & $68(43.31 \%)$ \\
\hline $\mathbf{1 0 .}$ & Piperacillin-tazobactum & $142(90.44 \%)$ & $15(9.55 \%)$ \\
\hline $\mathbf{1 1}$ & Meropenem & $144(91.71 \%)$ & $13(8.28 \%)$ \\
\hline
\end{tabular}


Higher rate of prevalence was shown in males $(56.68 \%)$ than females $(43.31 \%)$ in the present study which was comparable with study by SarojGolia et al., ${ }^{10}$ which also showed higher prevalence rate in males $(66.6 \%)$ than females $(33.3 \%)$, H. Ravi ChandraPrakash et al., ${ }^{11}$ males $(60.0 \%)$, females $(40.0 \%)$, Lakshmi et al., ${ }^{12}$ males (52\%), females $(48 \%)$.

Age wise distribution in the present study shows higher number of isolates from 41-60 years age group (38.21\%) followed by $>60$ years age group $(28.66 \%)$. Similar observation was made in study by SarojGolia ${ }^{10}$.

In the present study sample wise distribution shows higher number of isolates were obtained from pus, sputum and $22.48 \%$, $20.98 \%$ and $14.90 \%$.

This observation correlates with studies by SarojGolia et al., ${ }^{10}$, Lakshmi et al., ${ }^{12}$, Mohanasundaram et al., ${ }^{13}$, Arora ${ }^{14}$, Senthamarai S et al., ${ }^{15}$.

Antibiotic sensitivity testing in the present study shows higher sensitivity was exhibited by meropenem, piperacillin-tazobactum and amikacin. Increasing resistance was shown by cefixime, netilmicin and ceftriaxone. Similar sensitivity pattern was seen in study by Javiya et al., ${ }^{16}$ where sensitivity to imipenem was $78.57 \%$, meropenem $69.64 \%$. Study by Saroj Golia $^{10}$ shows higher sensitivity to piperacillin-tazobactum (91.07\%), imipenem (100\%), ceftazidime $(91.07 \%)$ which can be compared with the present study with sensitivity rate of $90.44 \%$ for piperacillintazobactum, $91.71 \%$ for meropenem but sensitivity to ceftazidime was slightly lower at $68.78 \%$. Another study by H. Ravichandra Prakash et al., ${ }^{11}$ exhibited least resistance to amikacin, piperacillin-tazobactum. This is in agreement with our study where in resistance to piperacillin-tazobactum was $9.55 \%$ and amikacin $14.64 \%$. Present study can also be compared with study by Sapna Mundheda et al., ${ }^{17}$ which showed sensitivity rate of piperacillin-tazobactum $(89.71 \%)$, imipenem (88.24\%), ceftazidime (61.76\%) which is in acceptance with our study.

Present study concludes that the prevalence rate of Pseudomonas aeruginosa was $18.3 \%$ from various clinical samples. Antibiotic profile shows higher sensitivity to carbapenems like meropenem, aminoglycosides like amikacin and combination drug like piperacillin-tazobactum rather than monotherapy. As Pseudomonas aeruginosa readily acquires resistance to antibiotics, there is need for judicious use of antibiotics with strict antibiotic policies, minimising the hospital stay and adherence of standard sterilisation practices.

\section{Acknowledgment}

We would like to express our sincere gratitude to Viswabharathi medical college and hospital, Head of the department of Microbiology, technical staff of Microbiology department.

\section{References}

1. Diagnostic Microbiology: Bailey and Scott's. $12^{\text {th }}$ edition.

2. Mackie and McCartney Practical Medical Microbiology. $14^{\text {th }}$ edition. Elsevier publication.

3. Medical Microbiology. A Guide to microbial infections. David Greenwood, Richard slack, Mike Barer, Will Irving. $18^{\text {th }}$ edition. Elsevier publications. $\begin{array}{llr}\text { 4. Agila Kumari } & & \text { Pragasam, } \\ \text { Balajiveeraraghavan, } & \text { E. Nalini, }\end{array}$ ShaliniAnandan, Keith S kaya. Indian J Med Microbiol 2018; 36: 303-16. 
5. Essentials of Medical Microbiology. ApurbaSankarSastry, SandhyaBhat K. $2^{\text {nd }}$ edition. Jaypee publishers.

6. Vikas Chandra yadav, Vepada Ravi kiran, Mahendra kumar jaiswal, Khileshwarsingh. A study of antibiotic sensitivity pattern of Pseudomonas aeruginosa isolated from a tertiary care hospital in south Chattisgarh. International $\mathrm{J}$ medical science and public health. 2017; 6 (3).

7. Ananthanarayan and panikers textbook of Microbiology $10^{\text {th }}$ edition. Universities Press.

8. Clinical and Laboratory Standards Institute (CLSI). 2014. Performance standards for antimicrobial susceptibility testing; Twenty fourth informational supplement. M 100-S24.

9. Chander Anil, Razamohammadshahid. 2013. Antimicrobial susceptibility patterns of Pseudomonas aeruginosa clinical isolates at a teriary care hospital in Kathmandu, Nepal. Asian J. Pharm. Clin.Res., vol 6, suppl. 3, 235-238.

10. SarojGolia, Suhani, Manasa S and Jyothi 2016.Isolation of Pseudomonas aeruginosa from various clinical isolates and its antimicrobial resistance pattern in a tertiary care hospital. Int J Curr. Microbiol. App. Sci. 5(3): 247-253.

11. Ravichandra Prakash H., Rashmi Belodu, Neena Karangate et al., 2012. Antimicrobial susceptibility pattern of Pseudomonas aeruginosa strains isolated from clinical sources.Journal of Pharmaceutical and Biomedical Sciences, 2012, 14 (05).

12. Renuga S, Lakshmi K, Chitralekha s, Illamani V. Prevalence of Pseudomonas aeruginosa and its susceptibility pattern in a tertiary care hospital. 2015. Int. J. Res. Pharm. Sci., 6(1), 27-30.

13. Mohanasundaram KM. The antibiotic resistance pattern in the clinical isolates of Pseudomonas aeruginosa in a tertiary care hospital; 2008-2010.ClinDiagn Res 2011; 5(3): 491-94.

14. Arora D, Jindal n, Kumar R, Romit emerging antibiotic resistance in Pseudomonas aeruginosa. Int J Pharm Sci 2011; 3(2): 82-4.

15. Senthamarai S, Suneel kumar reddy A, Sivashankar S et al., resistance pattern of Pseudomonas aeruginosa in a tertiary care hospital of kanchipuram, Tamilnadu, India. J. Clin. Diagn. Res 2014 may; 8(5): DC 30 - DC 32.

16. Javiya J.A, Ghatak S.B, Patel K.R, Patel J.A. Antibiotic susceptibility patterns of Pseudomonas aeruginosa at a tertiary care hospital in Gujarat, India. Ind. J. Pharmacol., 40 (5): 230-34.

17. SapnaMundheda, Anusharma, Kishore Ingole, Sadiya sheikh. Prevalence of Pseudomonas aeruginosa in surgical site infections in a tertiary care centre. Int $\mathbf{J}$ Curr. Microbiol App Sci (2017) 6 (4): 1202-1206.

\section{How to cite this article:}

Sarada Dasari, H. Ravichandra Prakash, C. Surya Kumari and Sisira, D. 2019. Prevalence of Pseudomonas aeruginosa in Various Clinical Samples and Its Antibiotic Susceptibility Pattern in a Tertiary Care Hospital. Int.J.Curr.Microbiol.App.Sci. 8(06): 1720-1724. doi: https://doi.org/10.20546/ijcmas.2019.806.205 